\title{
Elastic behavior of corrugated cardboard: Experiments and Modeling
}

\author{
Z. ABOURA ${ }^{\mathrm{a}}$, N. TALBI ${ }^{\mathrm{b}}$, S. ALLAOUI ${ }^{\mathrm{c}}$ \& M.L BENZEGGAGH ${ }^{\mathrm{c}}$ \\ ${ }^{a}$ L3M. IUT de Tremblay en France Paris 8 - \\ Rue de la Râperie 93290 Tremblay-en-France \\ ${ }^{\mathrm{b}}$ ESIEC Esp. Rolland Garros BP 102951686 Reims Cedex 2 \\ ${ }^{c}$ U.T.C Centre de Recherche Royallieu B.P 20529 F - 60205 Compiègne Cedex
}

\begin{abstract}
Motivated by computed homogeneous of linear corrugated cardboard behavior, an analytical model related to the assessment of equals behavior is proposed. This model takes into account the geometrical and mechanical properties of the corrugated cardboard constituents. An experimental methodology is also proposed to obtain both the in-plane elastic properties of each constituents and the corrugated cardboard. After model validation by a comparison with the experiment results, a parametric study is conducted studying the effect of geometrical parameters on in-plane elastic properties. Moreover, in order to assess the relevance of the homogenization method, a finite element model for 3 point bending test is created. Two approaches are adopted: the first one models separately the core and liner of the corrugated cardboard with thin shell element (3D approach), whereas the second approach being of particular interest in this work, considers the corrugated cardboard sandwich as a homogeneous plate and modeled therefore by a plate elements. It is shown that the simplified homogenized procedure is adequately accurate and ten time faster than the 3D approach for effectively analyzing corrugated cardboard panel in the preliminary and optimum design stages.
\end{abstract}

Key words : Corrugated cardboard, Experimental procedure, Homogenization, Finite element.

Corresponding author

E-mail address : aboura@utc.fr 


\section{Introduction}

"The packing must protect what it sells and must sell what it protects ", this citation summarizes the big functions of the packing that are divided in six principal families: paper, plastic, glass, metal, wood and composed material. The more used, the corrugated cardboard packing does not stop its increasing every year. This is due to its numerous advantages, notably the protection of the environment (completely recyclable) and its reduce coast. Nevertheless, its use in an optimum manner requires the knowledge of its mechanical behavior : elastic, inelastic, failure, etc...

The problem can be viewed by considering the corrugated cardboard as a structure and then be by norms relative to the composite or metallic sandwich structure. The main tests are 3 or 4-point bending (ASTM C393-62), as well as shear tests (ASTM C273-61). Norstrand et al [1] have evaluated the transverse shear stiffness of corrugated cardboard by ASTM block shear test and by three-point bending test. It has been found that the shear moduli determined by the bending test, are significantly lower than those obtained by the block shear test. Nevertheless, this approach doesn't allow to determine the stiffness matrix of such a material and makes therefore its homogenization for a finite elements calculation impossible. It is obvious that the possibility exists always to mesh the structure completely (skins and core) [ $2,3,4]$ leading to an extremely expensive process. In this study, an other alternative being well developed consists the replacing the corrugated cardboard by an equivalent orthotropic layer. This approach articulating around two points, will permit after homogenization to simplify the numerical calculations.

- The first point is to dedicate the different experimental protocols finalized for the sake of determination the mechanical properties of the constituents (skins and core) as well as those of the corrugated cardboard. The main difficulty resides in the experimental precautions in order to identify the necessary parameters in the absence of the pre-workbenches protocols.

- $\quad$ The second point is to dedicate the analytical modeling. Thus, by using the mechanical properties and geometrical parameters of the constituents (step of fluting, thickness of skins and core), this model should be able to predict the homogenized elastic behavior of the corrugated cardboard.

A comparison between the experimental observations, analytic model and finite elements prediction allows to judge the performances of the proposed model. It is accompanied by a 
parametrical study highlighting the effect of the structural parameters on some elastic properties.

\section{Experimental procedure}

\section{Material}

The corrugated cardboard is an orthotropic sandwich with the surface plies (facing) providing bending stiffness, separated by a lightweight corrugated core (fluting) that provides shear stiffness. The core and facing plates are glued along the edges of the facing plates to form a wide sandwich panel. Two main dirctions characterise this material. The first noted MD (machine direction) corresponds to the direction of manufacturing of the material. It coincides with the $x$ axis. The second noted CD (cross direction) corresponds to the transverse direction and coincides with the $y$ axis (Fig.1).

The cardboard is manufactured by SMURFIT company and is made from chemical Kraft fibres. The geometric material characteristics are presented in table 1.

Preparation of specimens is conducted for whole of tests, according to the recommendations of French norms NFQ 03-002 and NFQ 03-029.

\section{Tensile tests}

The used specimens are receptively inspired from ISO type NF T51-034 and NF Q03-002 on the mechanical characterization of plastic materials, paper and cardboard (Fig.2). Each specimen is instrumented by a bi-directional strain gage. The most important difficulty of the tensile tests on the corrugated cardboard resides in the bruising of the specimen heads at the griping time.

To increase the rigidity of the specimen heads, a polyester resin is injected between flutings and the cardboard skins to fill the existing emptiness. A particular precaution is taken at the time of the specimen preparation in the CD. As a matter of fact, the resin risks to flow in the useful part and distort the test.

With regard to the skins preparation, the specimen extremities are rigidified by impregnating in the resin. This technique allows consequently to avoid the recourse to the special bits recommended by the norm NF Q03-002. All specimens are conditioned at $23^{\circ} \mathrm{C}$ and $50 \% \mathrm{RH}$ for at least $24 \mathrm{~h}$ before testing. The tests are conducted under $3 \mathrm{~mm} / \mathrm{min}$ cross head speed using a sensitive load cell of $500 \mathrm{~N}$. 
A representative example of the normal stress-strain curve of the corrugated cardboard is pointed out in figure 3. This curve exhibits a linear part following by a non-linear one. The later seems to be governed by a damage phenomenon which is mainly localized on the skins. This phenomenon is more obvious in load-displacement curves of skin notably in the transverse direction of cellulose fibers (CD). It characterizes by small load falls (Fig. 3b). The obtained results under tensile tests are presented in table 2 . It is important to note that the result dispersion remains acceptable with a maximum value of $15.47 \%$ for $\mathrm{E}_{\mathrm{CD}}$. The mechanical properties are more important in the MD than $\mathrm{CD}$. One can conclude that these results are in an appropriate accordance with those results presented in the literature $[2,4,5]$.

\section{Shear tests}

The shearing tests represents a suitable manner to determine the two equivalent moduli of the transverse shearing: $G_{x z}$ and $G_{y z}$. Such tests are carried out according to ASTM 273-61. The following limits, relative to the geometry of test-tubes are to respect $h c<b / 2$ and $h c s<l / 12$ $[1,6]$ where $h c$ is the core thickness of the sandwich and $b$ and $l$ are respectively the width and the length of the specimen. The specimen is glued to loading blocks with a twocomponent epoxy glue (araldite) that cures 24 hours at room temperature (Fig.5). Then, it is conditioned at $23^{\circ} \mathrm{C}$ and $50 \% \mathrm{RH}$ for $24 \mathrm{~h}$. As far as the loading measurement is concerned a load cell of $20000 \mathrm{~N}$ load capacity is used.

The shear moduli are determined according to the ASTM C273-61 recommendation. The corresponding effective shear moduli in MD and CD are defined as follows :

$$
G_{i z}=\frac{\tau_{i z}}{\gamma_{i z}}=\frac{\theta H}{b \cdot l}
$$

$\mathrm{i}=\mathrm{x}, \mathrm{y}$

$\tau_{\mathrm{iz}}:$ shear stress

$\gamma_{\mathrm{iz}}:$ shear strain

$\theta$ : slope of the linear part of the load-displacement curve

$\mathrm{H}$ : total sandwich thickness

$\mathrm{b}:$ specimen width

1 : length of the specimen

The in-plane shear moduli of the skin and the corrugated are calculated using Baum's approximation [7] as follows 


$$
G_{x y}^{B}=0.387 \sqrt{E_{x} E_{y}}
$$

The measured values obtained from these tests are summarized in table 3. The dispersion concerning the out-plane shear remains acceptable (lower than 10\%).

Let's analyze the load evolution during the displacement for this test. In the case of MD, this evolution can be described by four phases before the failure (Fig. 6). The first stage is characterized by a linear shape (elastic phase). A first load fall is observed at the end of this stage corresponding to the downfall of flutings under shearing effect. Once the minimal fluting thickness reaches, the material enters in the third stage during which the load increases again. In this phase, the shear loading of fluting-skins interfaces takes place. The fourth stage represents a total damaging of the material. The glue of starch gives up and provokes the detachment of skins. With regard to the CD behavior, this later is characterized by an linear elastic phase before reaching the failure as the detachment of flutings (Fig. 7).

\section{Bending tests}

The specimens of the 3-points bending tests do not require any particular preparation. Their dimensions are $200 \times 60 \times 4.01 \mathrm{~mm}$. The span value is $160 \mathrm{~mm}$ giving the span-to-thickness ratio $(\mathrm{L} / \mathrm{h})$ equal to 39 minimizing therefore the shearing effect. The specimens are conditioned at $23^{\circ} \mathrm{C}$ and $50 \% \mathrm{RH}$ for at least $24 \mathrm{~h}$.

Beam deflection is measured by an LVDT transducer attached directly to the specimen. The overall capacity of this transducer is $50 \mathrm{~mm}$ and the load is measured by a sensitive load cell of $50 \mathrm{~N}$. The employed cross head speed is $2 \mathrm{~mm} / \mathrm{min}$.

The figure 8 presents a typical example of load-displacement in MD. Position and diameter of the load nose have a significant influence on the maximal value of the downfall load. However, the elastic behavior is practically the same under these experimental conditions. Hence, in the case of $10 \mathrm{~mm}$ load nose diameter positioned between two tops of undulation (case 1), the downfall load is less important and the loss of linearity is more pronounced in comparison with the case where the load nose is applied on the top of the undulation (case 2). This phenomenon can be explained by the apparition of a local distortion under the load noses on the superior skin. It is important to note that such distortion is less important in the second case. In order to minimize this phenomenon and to avoid the localization of the load in a particular point, other tests are carried out using $40 \mathrm{~mm}$ load nose diameter. The obtained results indicate an increase of the downfall load whereas the elasticity remains unchanged. 
The bending moduli presented in table 4 are obtained from the slope of the linear part of the load-displacement curve by using this relation :

$$
E=\frac{\Delta \cdot l^{3}}{4 \cdot b \cdot H^{3}}
$$

where
$\Delta:$ slope of the load displacement curve
1 : length between supports
$\mathrm{b}$ : width of the specimen
$\mathrm{H}$ : thickness of the specimen

The bending moduli are considerably close to the tensile moduli giving us a certain legitimacy to validate the tensile experimental procedure established for this material.

\section{Formulation of the analytical model}

To analyze the elastic behavior of the corrugated cardboard, the proposed technique is a pointwise lamination approach using the classical laminate theory. This approach is inspired from Ishikawa et al [8], Aboura [9, 10] and Scida et al. [11] related to the elastic behavior modeling of woven composites materials.

A unit cell representative of the corrugated cardboard is defined as in figure 9 and considered fthe skins and the undulated fluting as an assembling of many infinitesimal elements $d x$ of unidirectional lamina oriented at different angles.

The classical laminate theory is then applied to each element. The relationship between the inplane stress and moment resultants $N_{i}$ and $M_{i}$, and in-plane strains, curvature $\varepsilon_{j}$ and $\kappa_{j}$ is given by :

$$
\left\{\begin{array}{l}
N_{i} \\
M_{i}
\end{array}\right\}=\left[\begin{array}{ll}
A_{i j} & B_{i j} \\
B_{i j} & D_{i j}
\end{array}\right]\left\{\begin{array}{l}
\varepsilon_{j} \\
k_{j}
\end{array}\right\} \quad(i, j=1,2 \text { and } 6)
$$

in which $A_{i j}, B_{i j}$ and $D_{i j}$ are the in-plane stiffness for each infinitesimal element $d x$. These are defined by

$$
(A, B, D)=\int_{-h / 2}^{h: 2}\left(1, z, z^{2}\right) Q_{i j} d z
$$


in which

$$
Q_{i j}=\left[\begin{array}{ccc}
\frac{E_{x}}{1-v_{x y} v_{y x}} & \frac{v_{x y} E_{y}}{1-v_{x y} v_{y x}} & 0 \\
\frac{v_{y x} E_{x}}{1-v_{x y} v_{y x}} & \frac{E_{y}}{1-v_{x y} v_{y x}} & 0 \\
0 & 0 & G_{x y}
\end{array}\right](\mathrm{i}, \mathrm{j}=1,2,6)
$$

$Q_{i j}$ is evaluated for each constituent of the corrugated cardboard unit cell. This means that the superior and inferior skins and the fluting undulation are taken into account. The local stiffness of each infinitesimal element depends on the constituent elastic properties as well as on the fluting orientation defined by the local off-axis angle $\theta(x)$. This angle is calculated from the fluting median fiber function $H_{t}(x)$ :

$$
\theta(x)=\tan ^{-1}\left(\frac{d H t(x)}{d x}\right)
$$

$H_{t}(x)$ is assumed to be of sinusoidal form with a maximum thickness of $h c$ :

$$
H t(x)=\frac{h c}{2} \sin \left(2 \pi \frac{x}{P}\right)
$$

The existence of these angles leads to a reduction in the effective elastic moduli in $x$ and $y$ direction. Thus, the elastic constants for the fluting can be calculated as follows.

$$
\begin{aligned}
& E(\theta)_{x}=1 /\left(\frac{m^{4}}{E_{1}}+\left(\frac{1}{G_{12}}-\frac{2 v_{12}}{E_{1}}\right) m^{2} f^{2}+\frac{f^{4}}{E_{2}}\right) \\
& E(\theta)_{y}=1 /\left(\frac{f^{4}}{E_{1}}+\left(\frac{1}{G_{12}}-\frac{2 v_{12}}{E_{1}}\right) m^{2} f^{2}+\frac{m^{4}}{E_{2}}\right) \\
& v(\theta)_{x y}=E(\theta)_{x}\left(\frac{v_{12}}{E_{1}}\left(m^{4}+f^{4}\right)-\left(\frac{1}{E_{1}}+\frac{1}{E_{2}}-\frac{1}{G_{12}}\right) m^{2} f^{2}\right) \\
& G(\theta)_{x y}=1 /\left(2\left(\frac{2}{E_{1}}+\frac{2}{E_{2}}+\frac{4 v_{12}}{E_{1}}-\frac{1}{G_{12}}\right) m^{2} f^{2}+\frac{\left(f^{4}+m^{4}\right)}{G_{12}}\right)
\end{aligned}
$$


$m=\cos (\theta), f=\sin (\theta), E_{1}, E_{2}, v_{12}$ and $G_{12}$ are the elastic properties of the fluting and skins in the orthotropic plane.

Once the $Q_{i j}$ terms are calculated, the in-plane stiffness coefficients can be evaluated for each element in different regions of the unit cell by using equation (2).

The global matrices $\mathbf{A}_{\text {global }}, \mathbf{B}_{\text {global }}$ and $\mathbf{D}_{\text {global }}$ for the corrugated cardboard unit cell are calculated numerically from the local matrices A, B and $\mathbf{D}$ evaluated for each infinitesimal element with an average in the $x$ direction by

$$
(A, B, D)_{\text {globale }}=\frac{1}{P} \int_{0}^{P} A(x), B(x), D(x) d x
$$

From the global matrices $[\mathbf{A}],[\mathbf{B}]$ and $[\mathbf{D}]$, the effective Young's moduli, shear modulus and Poisson's ratio of the corrugated cardboard can be obtained as

$$
\begin{aligned}
& E_{x}=A_{11}^{*}-\frac{\left(A_{12}^{*}\right)^{2}}{A_{22}^{*}} \\
& E_{Y}=A_{22}^{*}-\frac{\left(A_{12}^{*}\right)^{2}}{A_{11}^{*}} \\
& v_{x y}=\frac{A_{12}^{*}}{A_{22}^{*}} \\
& G_{x y}=A_{66}^{*}
\end{aligned}
$$

Where $\left[\mathrm{A}^{*}\right]=[\mathrm{A}] / H, H$ is the total unit cell thickness.

The relation (8) remains valid only in the case of symmetric laminate, that means that the global matrix $[\mathbf{B}]$ is equal zero. The solution convergence depends on the integration step $d x$. Table 5 presents the global matrix [B] evolution of for three integration steps $d x: 0.1 \mathrm{~mm}$, $0.005 \mathrm{~mm}$ and $0.0005 \mathrm{~mm}$. One notes that from the second step of integration, the global matrix [B] approaches to zero. Therefore, the step integration $d x$ equal to $0.005 \mathrm{~mm}$ will be used for the model validation.

\section{Model validation}

In order to validate the analytical model, its predictions are compared with experimental results. The geometrical parameters of the unit cell are already summarized in Table 1. By using the mechanical properties of the skins and the fluting experimentally identified (Tab. 2 
and 3), the predicted values compared with the experimental results are obviously demonstrated in Table 6.

It is worth noting that the predictions describe fairly well the tensile and bending experimental results. The error remains lower than $5 \%$ for all values except for $E_{M D}$ and $v_{x y}$ where these errors are respectively $10.57 \%$ and $15.8 \%$. This difference can be explained by the sensitivity of the model to the geometrical parameters values.

The evaluation of the impact of these geometrical parameters on the numerical results is performed by simulation. In fact, the elastic properties variation according to the step of the undulation, the corrugated thickness and the skin thickness are first determined. The predictions are then well demonstrated as in figures 10,11 and 12 . It is obvious that the variation of the step undulation has a little influence on the $\mathrm{E}_{\mathrm{MD}}$ modulus. However, the other moduli are strongly influenced by this parameter. So, a gap of $20 \%$ on this parameter can provoke an error of $7.5 \%$ on the $\mathrm{E}_{\mathrm{CD}}$ modulus. This influence is even more perceptible concerning the curves in figures 11 and 12. Moreover, a variation of $20 \%$ of the total thickness drags an error between 5\% and $15 \%$ related to the elastic parameters. A variation of $10 \%$ on skin thickness leads to a variation of $10 \%$ on the Young and shear modulus . Note that $10 \%$ on skin thickness variation represents $0.016 \mathrm{~mm}$, therefore a particular attention should be taken during the measure of this parameter.

Figures 13 and 14 summarize the combined effect of the thickness of the corrugated and undulation step on the evolution of the elastic properties of the material obtained by the analytical model. The geometrical characteristics of the micro fluting and middle fluting are fixed like limits of the interval (thickness: $\mathrm{H} 1=1.55 \mathrm{~mm}$ for micro fluting and $\mathrm{H} 2=4.01 \mathrm{~mm}$ for middle fluting, and the undulation step: $\mathrm{P} 1=3.26$ for micro fluting and $\mathrm{P} 2=8 \mathrm{~mm}$ for middle fluting).

\section{- Effect on membrane properties}

The first observation concerns the influence of the global thickness of the corrugated cardboard. The $\mathrm{E}_{\mathrm{x}}$ modulus $\left(\mathrm{E}_{\mathrm{MD}}\right)$ is strongly influenced. Actually, for a constant undulation step, the fall is about $60 \%$ (2400 MPa for a thickness of $1.5 \mathrm{~mm}$ against $1000 \mathrm{MPa}$ for a thickness of $4 \mathrm{~mm}$ ). The $\mathrm{E}_{\mathrm{y}}$ modulus $\left(\mathrm{E}_{\mathrm{CD}}\right.$ ) is less affected. It displays a fall of $45 \%$ (of 1100 $\mathrm{MPa}$ for $1.5 \mathrm{~mm}$ to $600 \mathrm{MPa}$ for $4 \mathrm{~mm}$ ).

With regard to the fluting step influence, it appears that the modulus $E_{x}$ remains almost insensible to this value whereas the value of $\mathrm{E}_{\mathrm{y}}$ modulus increases about $40 \%$ when the fluting step decreases from $8 \mathrm{~mm}$ to $3 \mathrm{~mm}$. The influence of the thickness remains perceptible on the 
in-plane shear modulus $\mathrm{G}_{\mathrm{xy}}$. An increase of $62.5 \%$ of the thickness induces a decrease about $50 \%$ of the $\mathrm{G}_{\mathrm{xy}}$ modulus.

The effect of the fluting step is more important for the strong thickness ( $4 \mathrm{~mm})$. The modulus $\mathrm{G}_{\mathrm{xy}}$ value decreases by $25 \%$ when the step evolves from $3 \mathrm{~mm}$ to $8 \mathrm{~mm}$. Whereas, this fall is only $6 \%$ for $1.5 \mathrm{~mm}$ thickness situation.

\section{- Effect on bending properties}

The bending rigidities are more sensitive to the thickness than the membrane properties (Figures 16 and 17). Thus, whatever the undulation step, the fall of the bending rigidities $\mathrm{D}_{11}$ and $\mathrm{D}_{22}$ is practically $90 \%$ when the thickness varies from $4 \mathrm{~mm}$ to $1.5 \mathrm{~mm}$. On the other hand, as for the elastic modulus $E_{x}$, the bending rigidity $D_{11}$ remains insensible to the step undulation except for $4 \mathrm{~mm}$ thickness. Indeed, a fall of $15 \%$ is calculated when the step undulation increase from 3 to $5 \mathrm{~mm}$ followed by a constants values until a step of $8 \mathrm{~mm}$.

The bending rigidity in cross direction exhibits an other behaviour. In fact, the influence of the step undulation is important for $4 \mathrm{~mm}$ thickness. The $\mathrm{D}_{22}$ fall by $56 \%$ when the fluting step increases from $3 \mathrm{~mm}$ to $8 \mathrm{~mm}$. This influence remains perceptible for higher thickness (4 $\mathrm{mm}$ to $3 \mathrm{~mm}$ ). However the $\mathrm{CD}$ bending rigidity, for the lower thickness, are not influenced by the step undulation.

This parametric study shows an antagonist evolution of membrane and bending rigidities versus total thickness. Nevertheless, the fluting step affect the $E_{y}$ and $G_{x y}$ more than the other parameters. The highest values are obtained for the lowest step undulation. Thus, the choice of geometrical parameters for optimal elastic parameters depends on the nature of loading. For a complex loading, a compromise between membrane and bending rigidities is necessary. This analytical model seems to be an interesting tool for making decision regarding the geometrical parameters choice for an optimum elastic solution.

\section{Finite element analysis}

The main advantages of the mechanical behavior simulating of corrugated cardboard product are:

- decreased development costs, since less experiments are necessary in the process development phase;

- a faster development phase, since more information on the mechanical properties of a corrugated cardboard is obtained in an early stage of its design; 
- the possibility of testing packages made from new corrugated cardboard materials, even if these materials have to be developed;

- being able to study the local phenomena (as local stresses and displacements).

In order to assess the relevance of the developed homogenization method, a finite element model is created for the 3-point bending. The geometrical characteristics of this model are chosen similar to those used in the corresponding experiments.

The used element is the basic triangular 3-nodes flat shell. It's a combination of CST plane stress and DKT plate bending elements [12,13], based on the Kirchhoff hypotheses under a discreet manner and proposed by Dhatt [14] and Stricklin et al. [15] for the linear analysis of thin plates. The stiffness matrix integration is performed by using three point Gauss quadrature rule in area coordinates. To determine the element stiffness matrix in local coordinates of a particular element, the global coordinate of each node in this element is transformed into the local coordinate. The stiffness matrix in local coordinates is then transformed into corresponding global coordinates. A transformation is required for this purpose which is discussed in detail in Zienkiewicz [16]. The DKT facet shell element seems to be the best element of its category while taking into account the aspects: simplicity in the calculations, efficiencies in the displacement and stress estimations and results reliability.

The FE program Reflex-v.1 [17] is used to calculate and GID-v.7 to model the actual corrugated cardboard sandwich panel. Two approaches are adopted:

- Shell approach (3D) which model separately the core and liner (skin) of the corrugated cardboard with thin shell element (Fig. 18). It is an association of two triangular elements (DKT plate element for bending and classical CST membrane). The adhesive between the liner (skin) and medium (core or undulation) is modeled as a perfect bonding. As a matter of fact, the extreme positions of the medium are connected directly to the liner by sharing the same node. Gilchrist et al. [3] are modeled the corrugated board by this method and a second one. In this later, a multi-point constraint (MPC) technique is utilized. The MPC chosen acts as a beam element between the two nodes that connects the liner and the medium and imposes the same rotations and translation on each nodes. They find, in all of the two models, that the predictions are almost the same. However, such an approach is bound to have a high number of elements and degrees of freedom which increase computational coast when one is interested in the overall response of the structure. In the CD model, 900 elements and 1070 nodes are required. The skin and flute elastic properties are assumed to be the same and are presented in table 2. 
- Plate approach (2D) considers the corrugated cardboard sandwich as a homogeneous plate and is modeled therefore by a triangular 3-node DKT plate element. For this model, the effect of the mesh refining on the solution convergence is investigated. Thus, 80, 160 and 240 elements are used. The elastic properties introduced in the FE analysis are those obtained by the analytical model and presented in table 6 .

First, let's analyze the effect of the number of elements on the 2D model. Refining the mesh has not a significant influence on the result accuracy (Table. 7). For this reason, the 2D model with 160 elements is considered for analysis after mesh convergent studies.

Table 8 presents a comparison between experimental and the two FE approaches results. The calculation times (CPU) of personnel computer with AMD Duron $850 \mathrm{MHz}$ processor are also indicated for the two approaches. The ratio FE / experiment for the $3 \mathrm{D}$ approach is 1.07. These same ratios are 1.08 and 0.88 respectively for $\mathrm{MD}$ and $\mathrm{CD}$ for the simplified homogenization procedure. The correlation between experimental and the two FE approaches is correct. However, for an overall behavior, the time calculation of the $2 \mathrm{D}$ approach is 10 time lower than that of the 3D approach. It appears that the simplified homogenization procedure is adequately accurate and fast for effectively analyzing corrugated cardboard panel in the preliminary and optimization design stages.

\section{Conclusions}

Although the corrugated cardboard has a constitution of a sandwich structure, its structural properties are revealed once the packing structure is constituted. This fact leads to consider that this sandwich is like a monolithic material. Hence, an experimental protocol is developed to determine its $2 \mathrm{D}$ orthotropic stiffness matrix.

As far as the modeling is concerned, an analytical model, taking into account the geometrical and mechanical properties of the cardboard constituents, is also proposed to predict these inplane properties. This model is also able to predict the bending properties.

After the model validation showing a good correlation between experimental and analytical results, a parametric study is conducted. This allows to analyze the effect of geometrical parameters on in-plane elastic properties. Thus, it appears that the $\mathrm{E}_{\mathrm{MD}}$ modulus and bending properties $\left(D_{11}\right.$ and $\left.D_{22}\right)$ are strongly influenced by the total thickness of the corrugated whereas the $\mathrm{E}_{\mathrm{CD}}$ modulus is more affected by fluting step. The proposed model should be an interesting tool for a help to decision on the choice of geometrical parameters for an optimum elastic solution 
In order to assess the relevance of the homogenization method developed for this purpose, a finite element analysis is used. The main goal of this work is to verify the possibility to use a 2D meshing of the corrugated cardboard, in stead of of a 3D meshing extensively used in the literature. It was found that the simplified homogenization procedure is adequately accurate and fast for effectively analyzing corrugated cardboard panel in the preliminary and optimum design stages.

\section{References}

1 Nordstrand T.M. and Carlsson L.A, Evaluation of transverse shear stiffness of structural core sandwich plates Composite structures 1997, 37,145-153.

2 Patel P, Nordstrand T.M, and Carlsson L.A, Local buckling and collapse of corrugated cardboard under biaxial stress Composite structures 1997, 39, 93-110.

3 Gilchrist A.C, Suhling J.C and Urbanik T.J, Nonlinear finite element modeling of corrugated cardboard Mech.of Cellulosic Mat. 1999, 85, 101-107.

4 Nordstrand T.M, Parametric study of the post-buckling strength of structural core sandwich panels Composite structures 1995, 30, 441-451

5 El Damatty A.A.,. Mikhael A and Awad A.A, Finite element modelling and analysis of a cardcardboard shelter Thin-Walled Structures 2000, 38, 145-165.

6 Desrumaux F. Contribution à l'analyse micromécanique du comportement élastique en dommageable de composites monolithiques et sandwichs, intégration dans un code de calculs par éléments finis Thèse de doctorat Université de technologie de Compiègne 2000.

7 Baum G.A., Brennan D.C. and Haberger C.C. Orthotropic elastic constants of paper TAPPI J 1981, 64, 97-101

8 Ishikawa T, Chou TW. Stiffness and strength behavior of woven fabric composites. Jour. of Mat. Sci.. 1982, 17, 3211-3220.

9 Aboura, Z., Etude du processus de délaminage Mode I, Mode II et Mode mixte (I et II) de matériaux composites à renforts tissés à différentes vitesses de sollicitation, Thèse de Doctorat, Université de Technologie de Compiègne 1993.

10 Aboura, Z., Chouchaoui, C.S. and Benzeggagh, M.L., Analytical model of woven composite laminate. Superposition effect of two plies, ECCM 6, 1993, Bordeaux. 
11 Scida, D., Aboura, Z., Benzeggagh, M.L. and Bocherens, E., Prediction of elastic behaviour of hybrid and non-hybrid woven composite, Composites, science and technology. 1997, 57, 1727-1740

12 Batoz J.L, Bath K.J, and Ho L.W, A Search for the Optimum Three-Node Triangular Plate Bending Element. Rapport 82448-8, Massachusetts Institute of Technology, December 1978.

13 Batoz J.L, Bath K.J,.and Ho L.W, A study of Three-Node Triangular Plate Bending Elements, Int. J. Num. Meth. In Eng.,. 15, 1771-1812,.

14 Dhatt G., Numerical Analysis of Thin Shells by Curved Triangular Elements Based on Discrete Kirchhoff Hypothesis, Proc. ASCE Symp. On Applications of FEM in Civil Engineering, Vanderbit University, Nashville, Tenn. Nov. 1969, 13-14.

15 Stricklin J.A., Haisler W., Tisdale P. and Gunderson R., A rapidly Converging Triangular Plate Element, AIAA J1969., 7, 180-181.

16 .Zienkiewicz O.C. and Taylo r R.L, The Finite Element Method, $4^{\text {th }}$ Ed. McGraw-Hill, New York, 1991.

17 Batoz J.L and Dhatt G, Modélisation des structures par éléments finis, Volume 1: Solides élastiques. Hermèse Edition 1992 


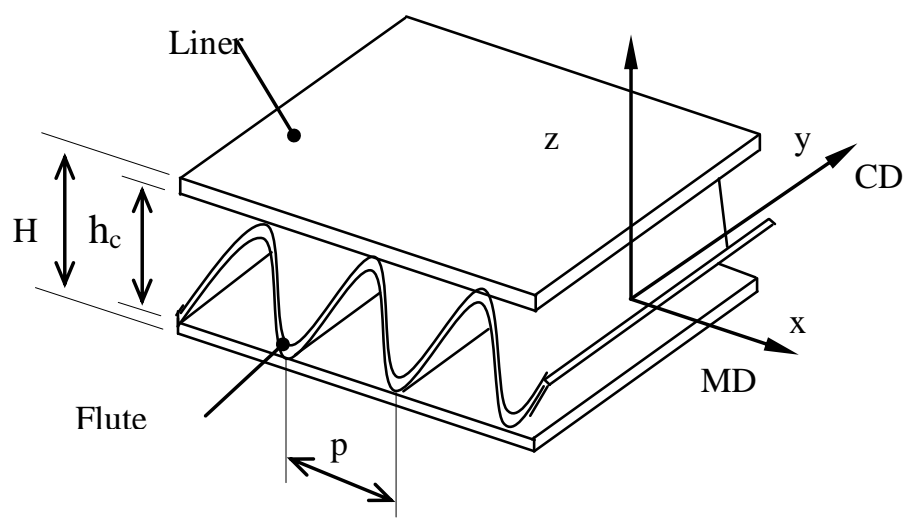

Figure 1: Cardboard panel geometry

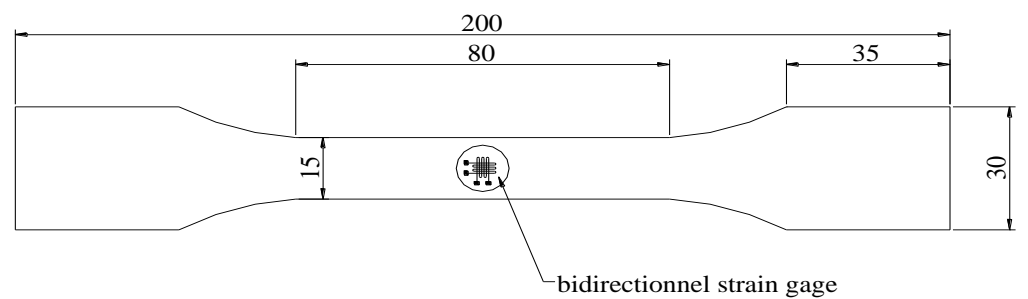

Figure 2 : Tensile specimen

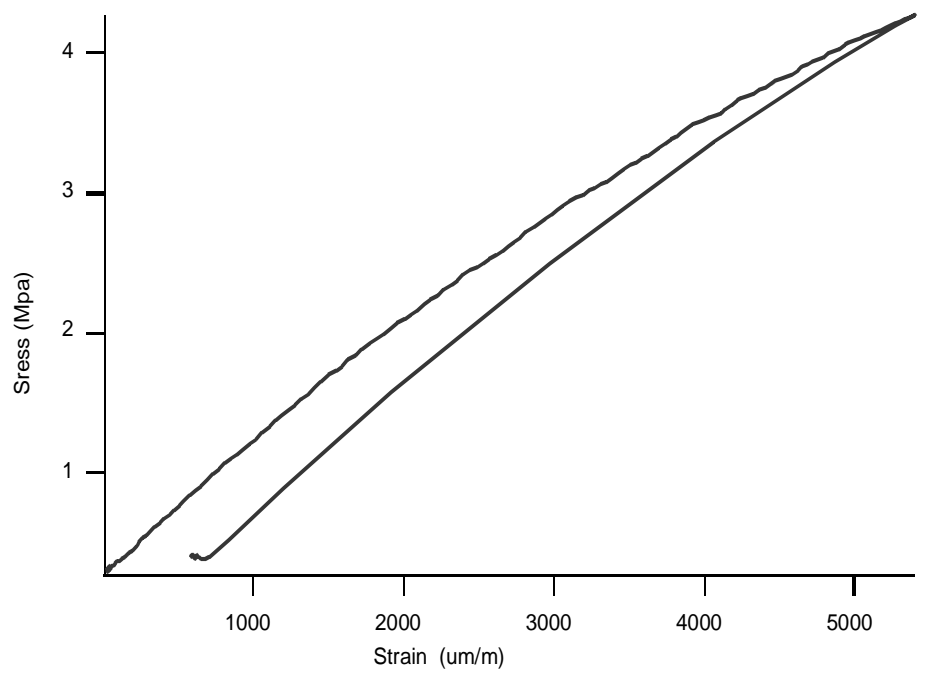

Figure 3 : Cardboard corrugated stress-strain tensile curve. 


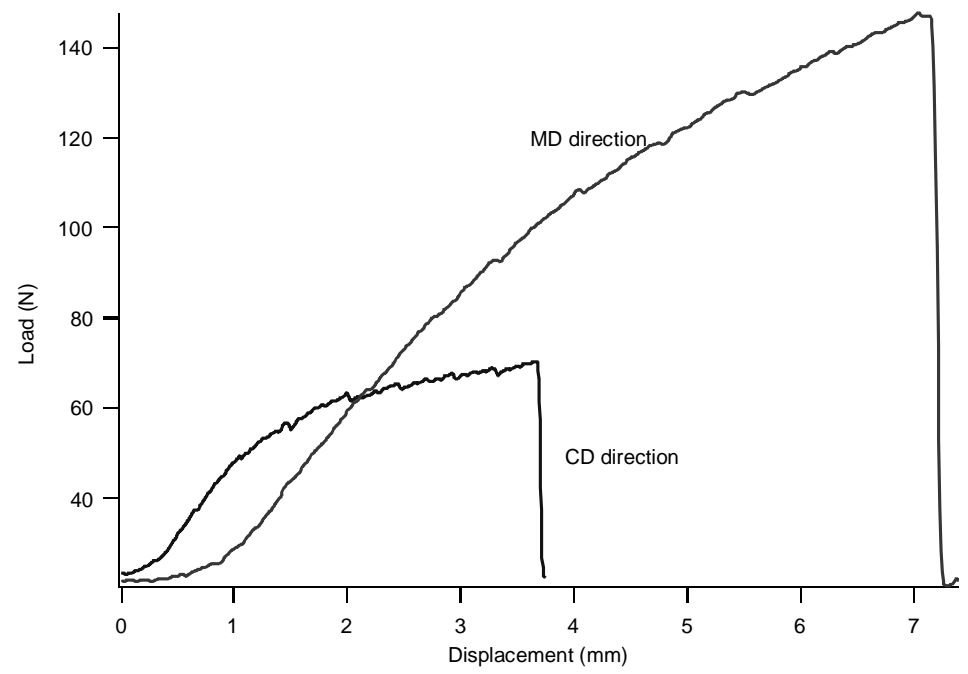

Figure 4 : Skin load displacement tensile curve

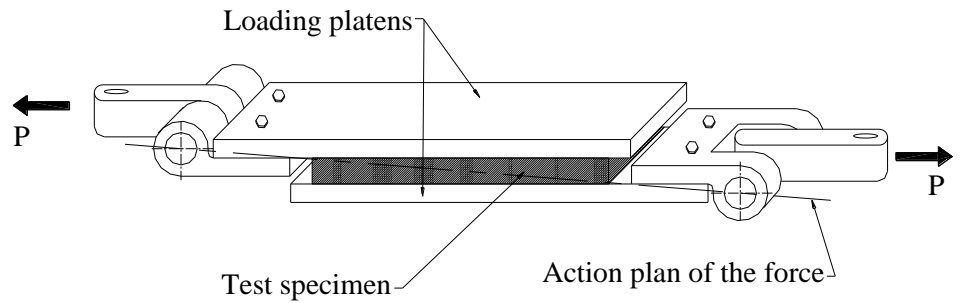

Figure 5 : ASTM block shear test 


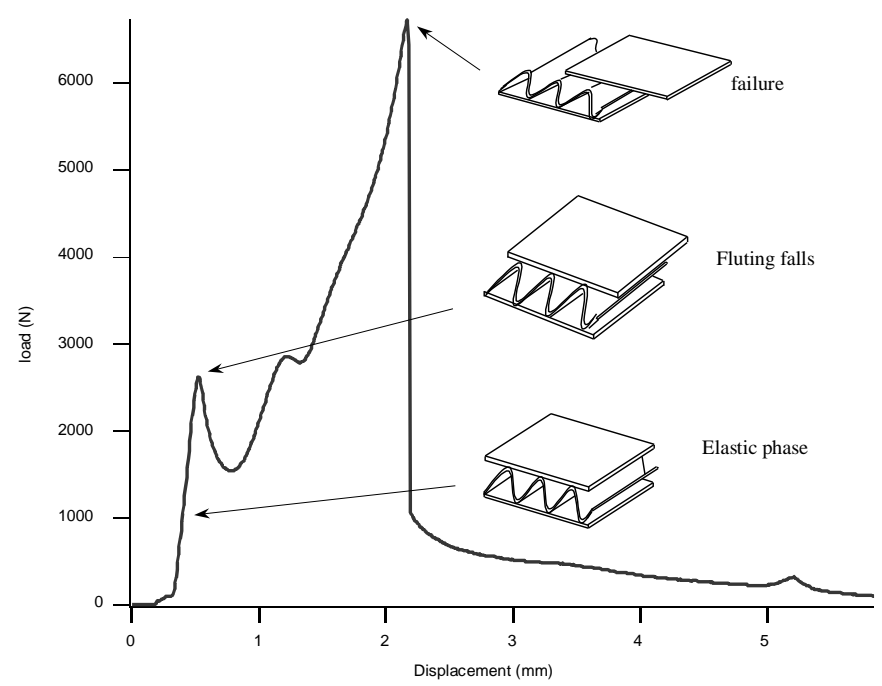

Figure 6 : MD load-displacement shear curve.

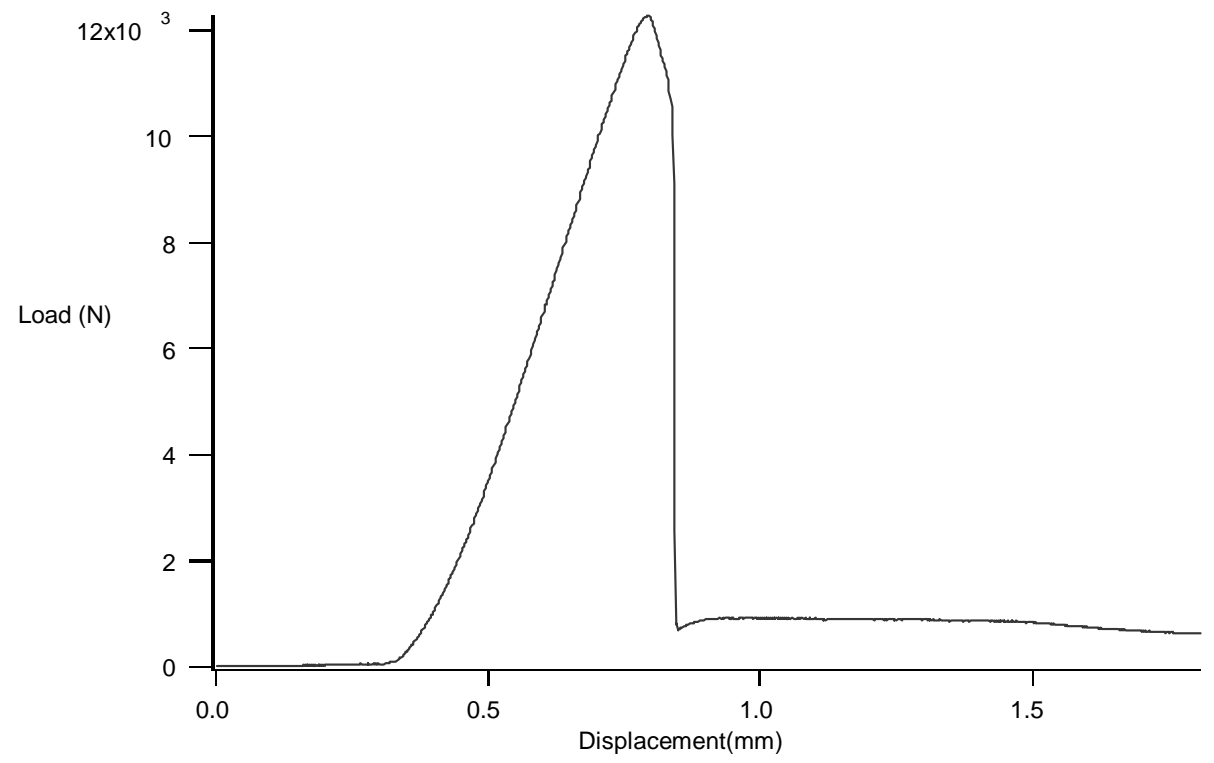

Figure 7 : CD load-displacement shear curve. 


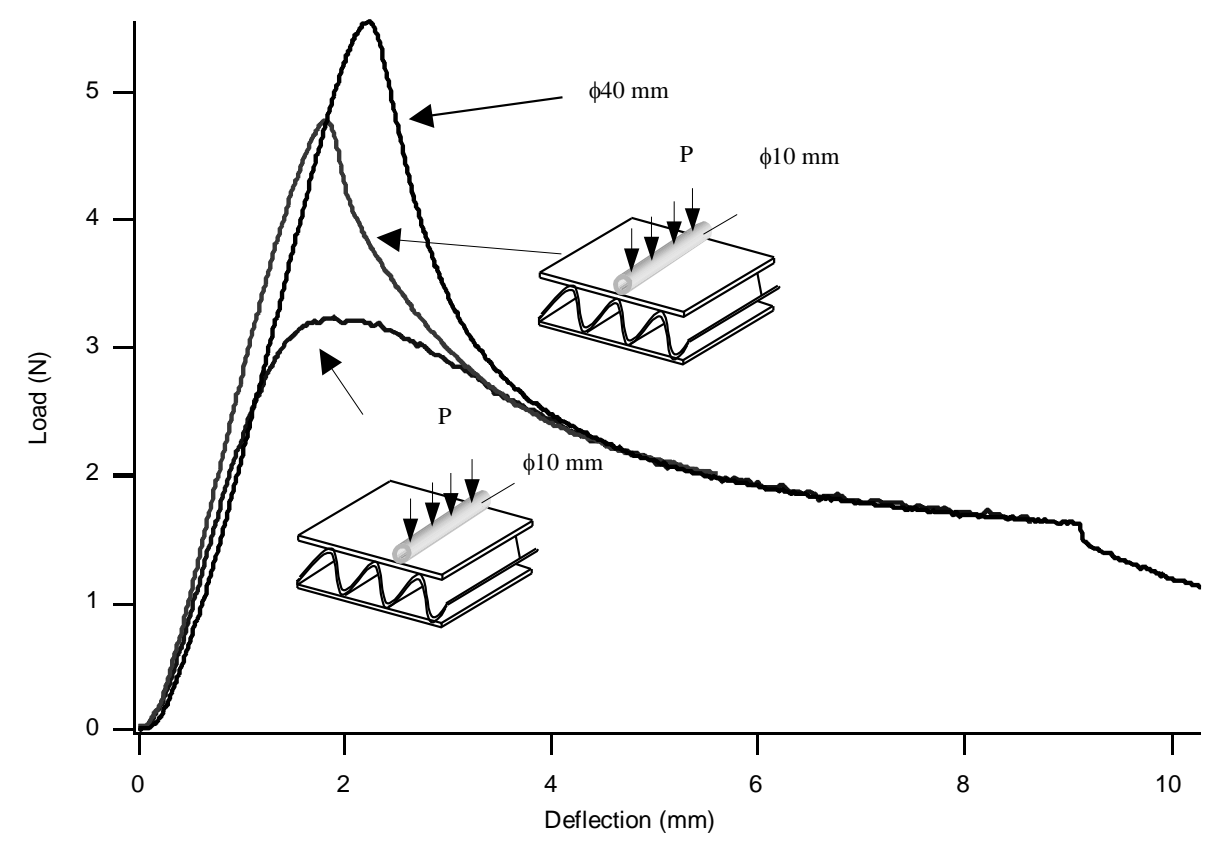

Figure 8 : MD load-deflection curves for different load nose diameters.

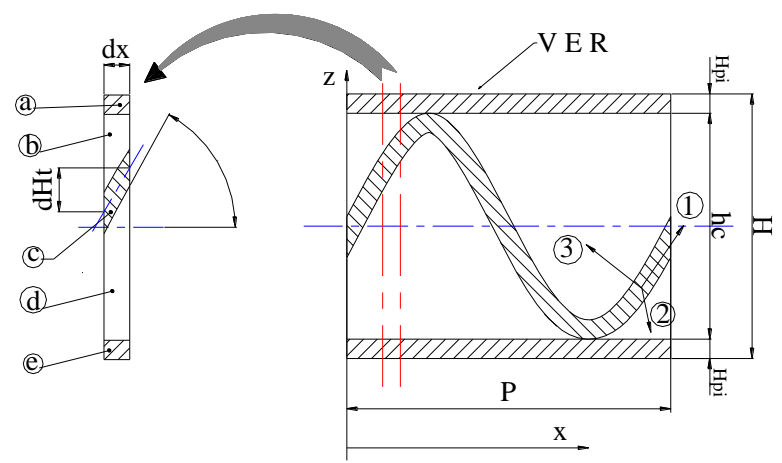

(a) superior skin

(b) emptiness

(c) fluting

(d) emptiness

(e) inferior skin

Figure 9 : Unit cell representative of the corrugated cardboard 


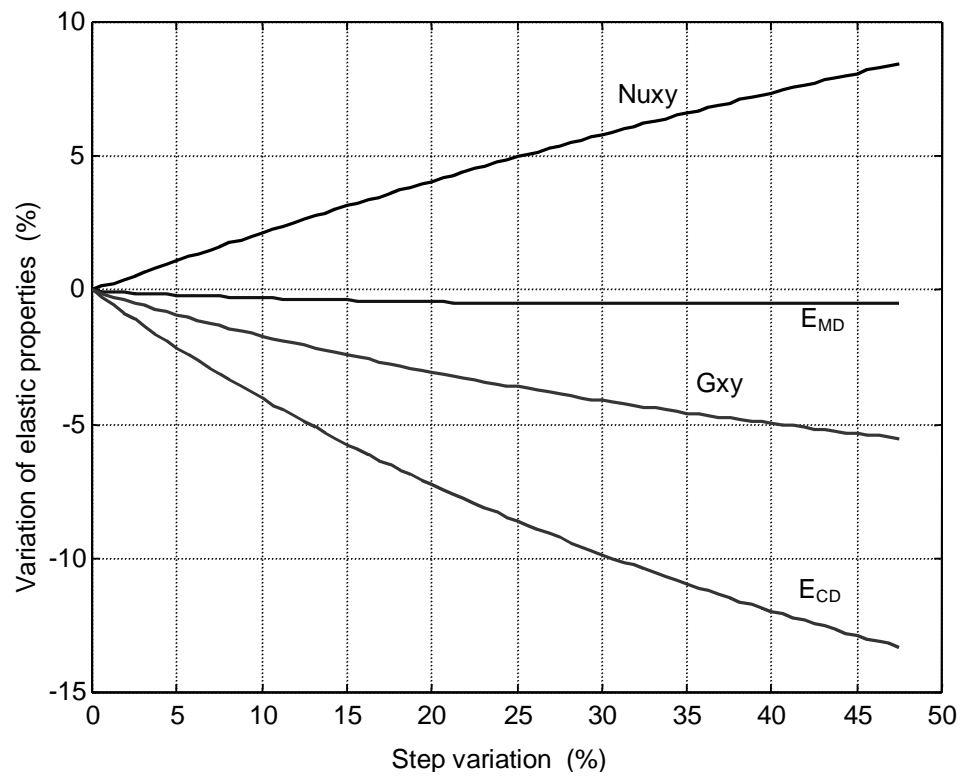

Figure 10 : Variation of elastic properties versus step variation.

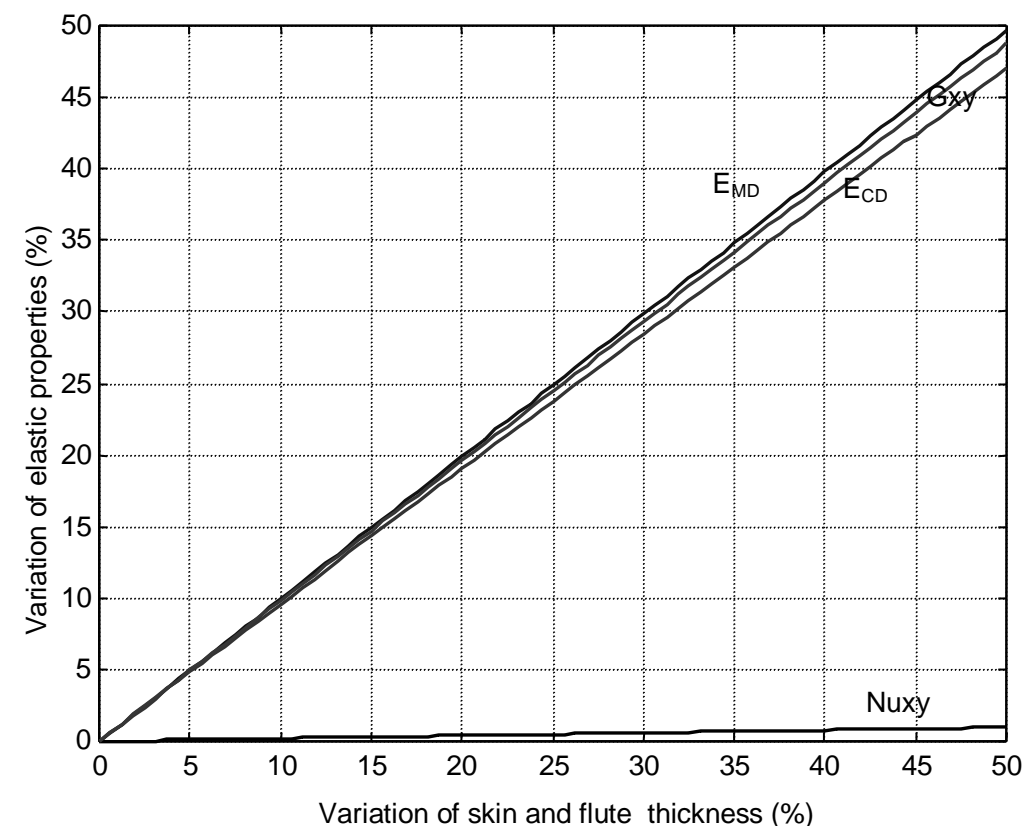

Figure 11 : Variation of elastic properties versus skin and flute thickness variation. 


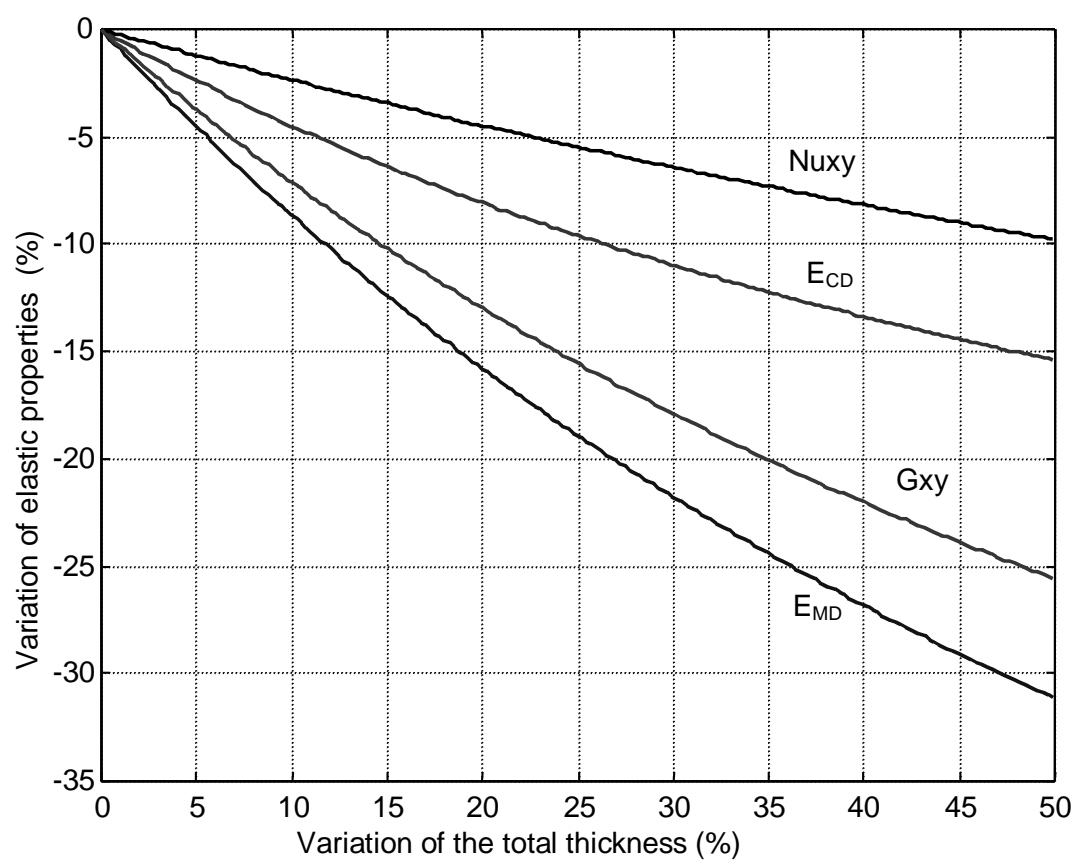

Figure 12 : Variation of elastic properties versus total thickness variation.

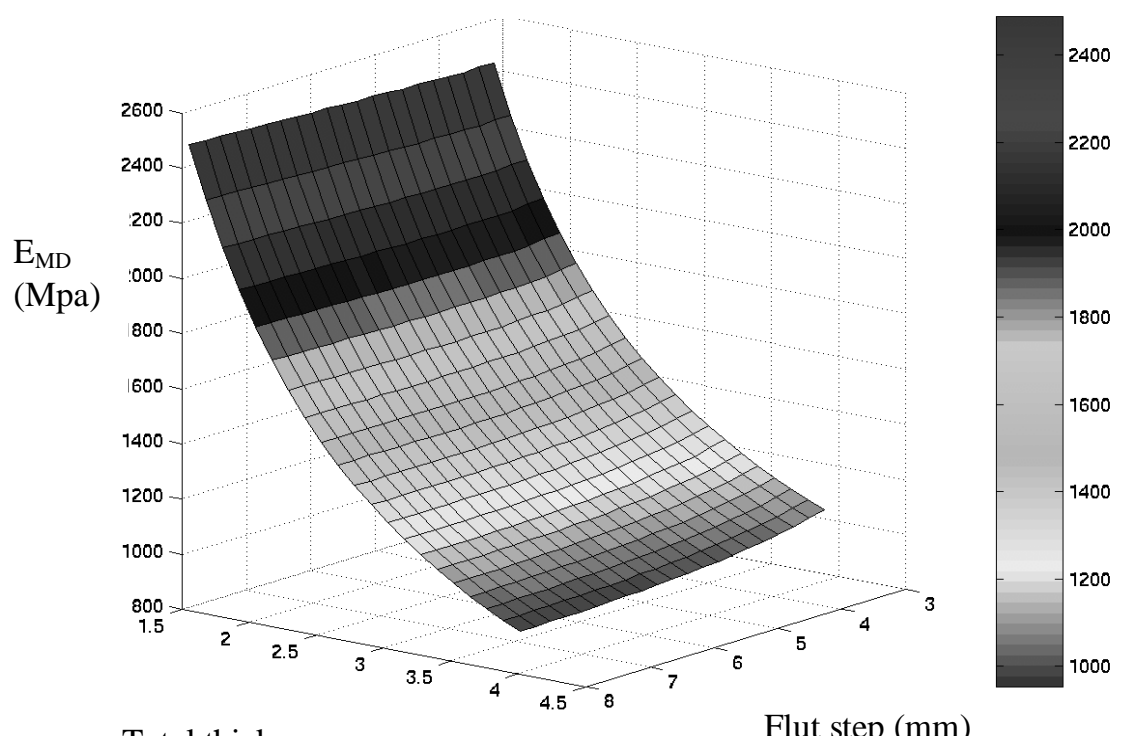

Total thickness

Flut step (mm)

Figure 13 : Evolution of $\mathrm{E}_{\mathrm{MD}}$ versus thickness and flute step 


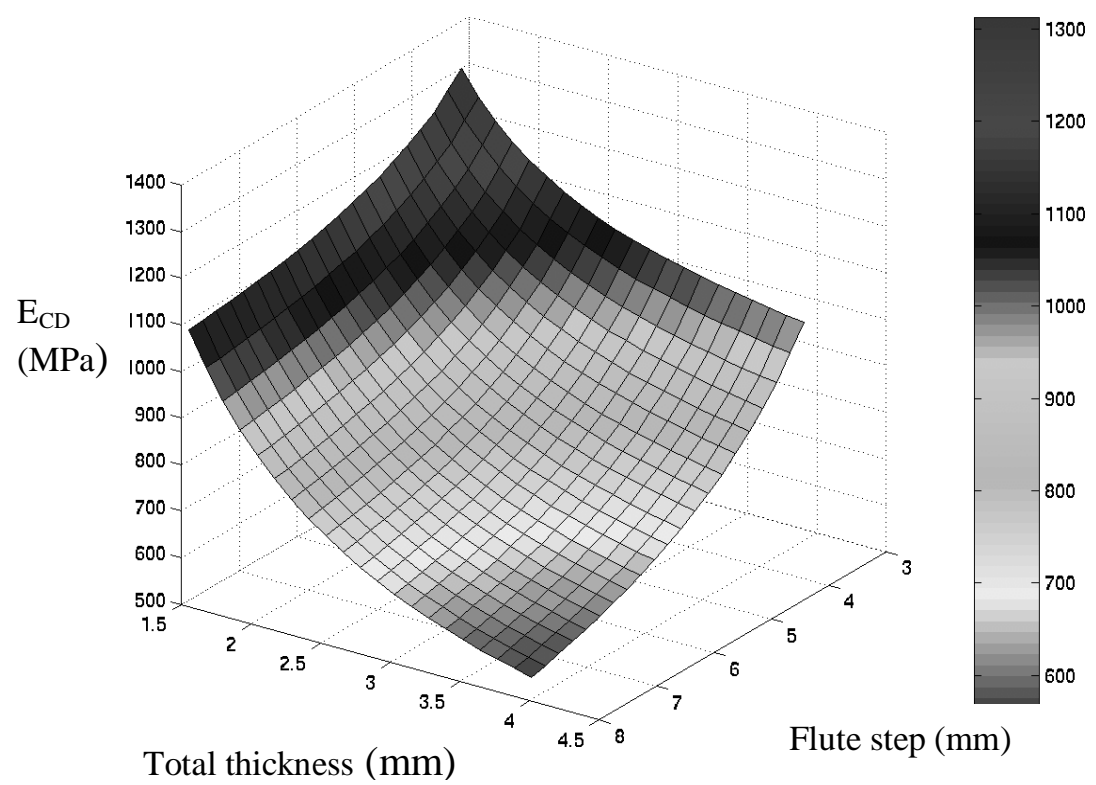

Figure 14 : Evolution of $\mathrm{E}_{\mathrm{CD}}$ versus thickness and flute step 


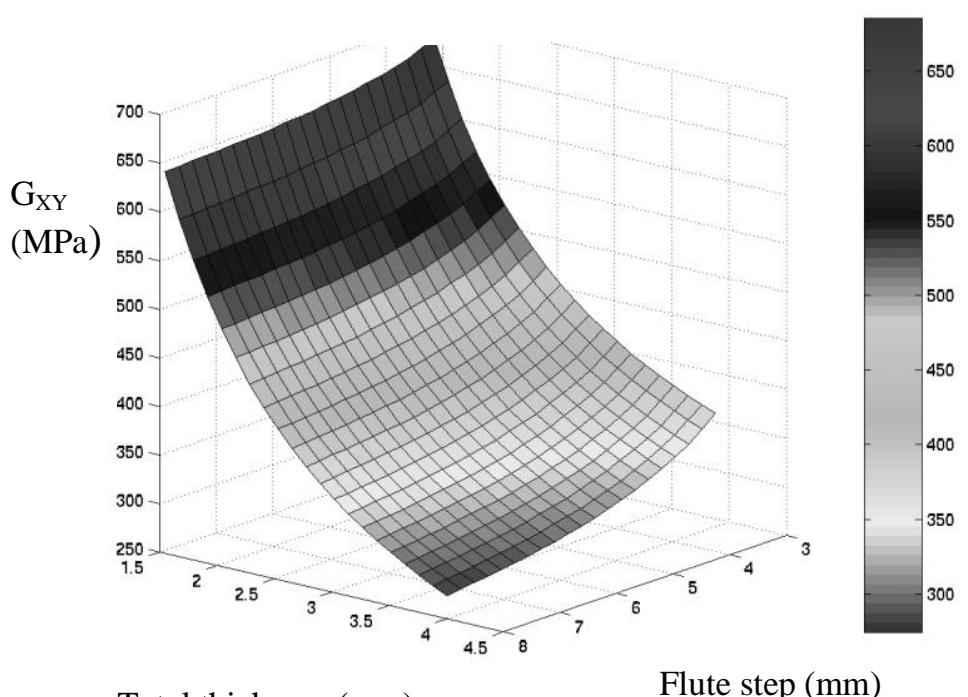

Total thickness (mm)

Flute step (mm)

Figure 15 : Evolution of $G_{x y}$ versus thickness and flute step

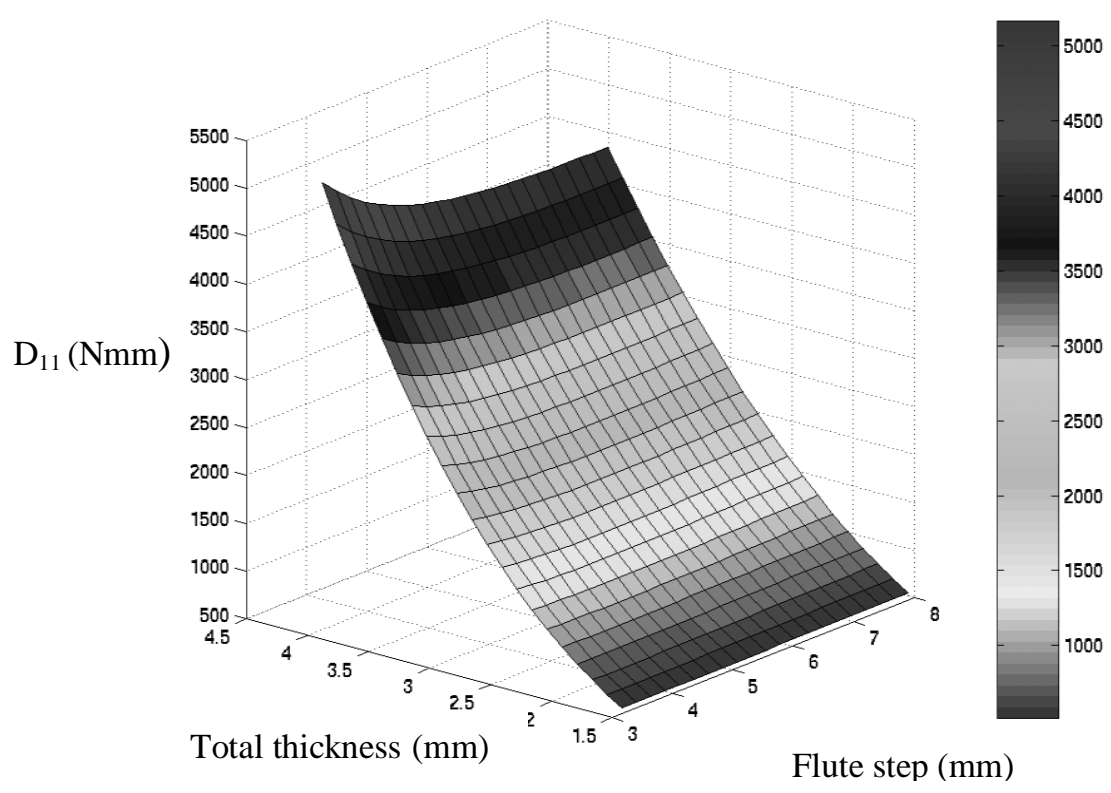

Figure 16 : Evolution of bending rigidity D11(MD)versus thickness and flute step 


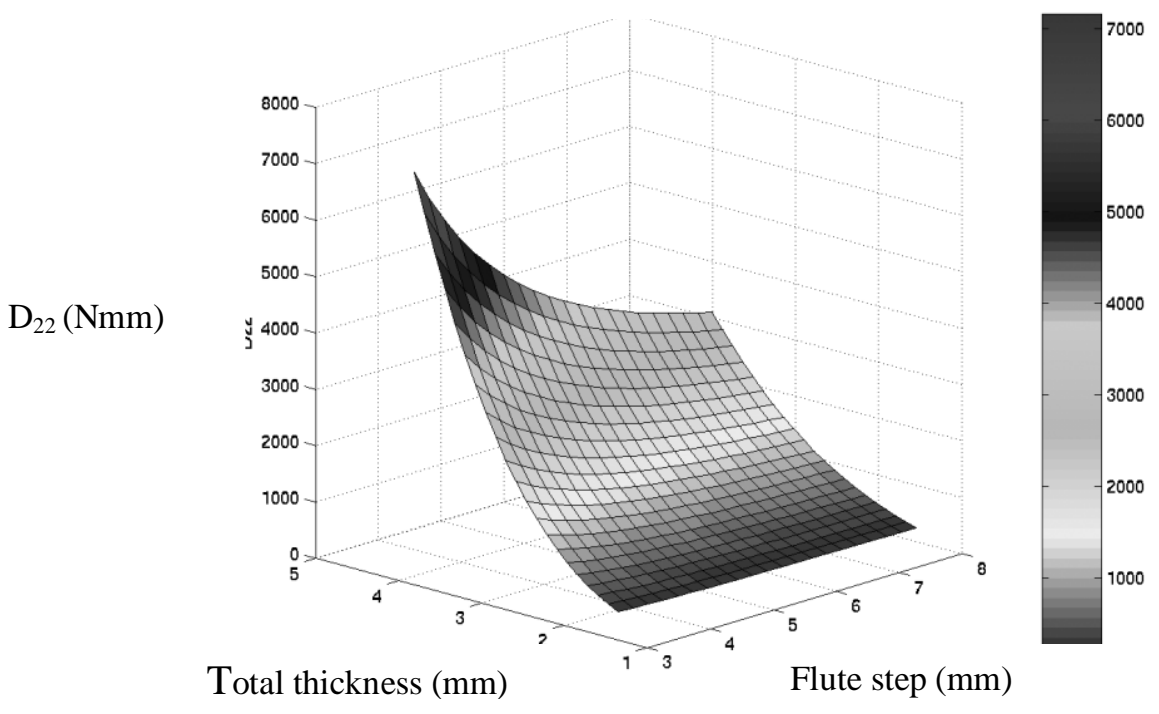

Figure 17 : Evolution of bending rigidity $\mathrm{D}_{22}(\mathrm{CD})$ versus thickness and flute step

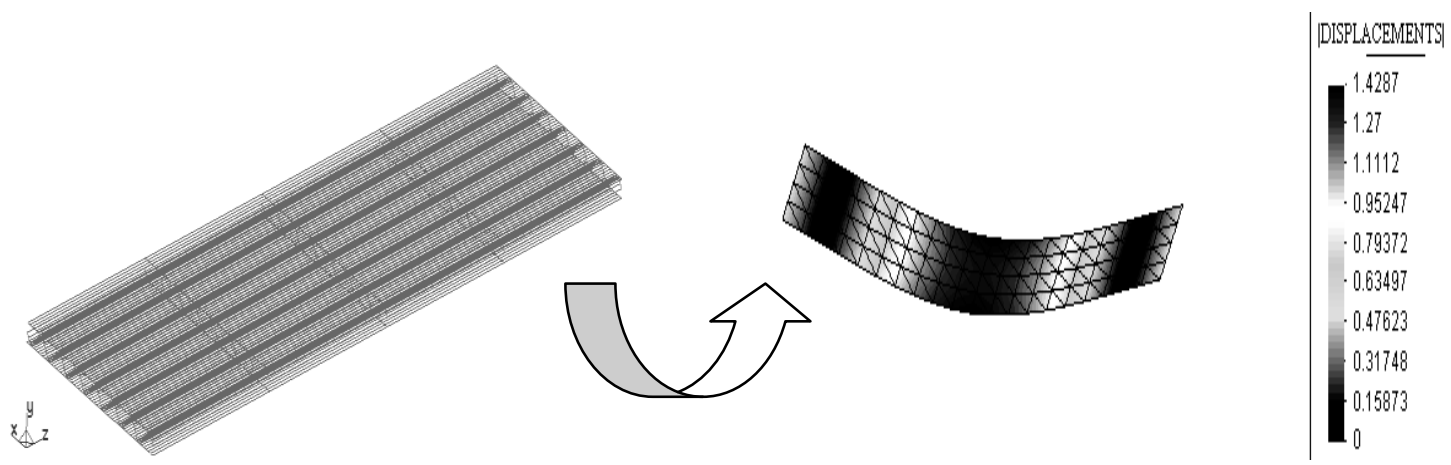

Figure $18: 3 \mathrm{D}$ and 2D finite elements meshing. 


\begin{tabular}{cccc}
\hline Thickness(mm) & Flute step (mm) & Liner thickness (mm) & Nbre of flute by meter \\
\hline 4.01 & 8 & 0.16 & 125 \\
\hline
\end{tabular}

Table 1 : Geometrical parameters

\begin{tabular}{ccccccc}
\hline & $E_{M D}(\mathrm{Mpa})$ & $v_{x y}$ & $\sigma_{M D} \square(M p a)$ & $E_{C D}(M p a)$ & $v_{y x}$ & $\sigma_{C D} \square(M p a)$ \\
\hline Skins & $8258.53 \pm 744.03$ & $0.39 \pm 0.061$ & $50.07 \pm 1.60$ & $3406.84 \pm 258.4$ & $0.14 \pm 0.01$ & $23.51 \pm 0.71$ \\
Corrugated & $863.05 \pm 99.50$ & $0.27 \pm 0.03$ & $4.44 \pm 0.14$ & $554.97 \pm 85.89$ & $0.22 \pm 0.03$ & $2.760 \pm 0.28$ \\
& & & & & & \\
\hline
\end{tabular}

Table 2 : Tensile properties of skins and cardboard corrugated.

\begin{tabular}{cccc}
\hline $\begin{array}{c}\text { Gxz Corrugated } \\
(\mathrm{Mpa})\end{array}$ & $\begin{array}{c}\mathrm{G}_{\mathrm{yz}} \text { Corrugated } \\
(\mathrm{Mpa})\end{array}$ & $\begin{array}{c}G_{x y}^{B} \text { Skins } \\
(\mathrm{Mpa})\end{array}$ & $\begin{array}{c}G_{x y}^{B} \\
\text { Corrugated } \\
(\mathrm{Mpa})\end{array}$ \\
\hline $2.32 \pm 0.12$ & $3.926 \pm 0.14$ & 2052.76 & 267.83 \\
\hline
\end{tabular}

Table 3 : Shear properties of cardboard corrugated and skins

\begin{tabular}{cccc}
\hline $\mathrm{E}_{\mathrm{MD}}(\mathrm{MPa})$ & $\mathrm{E}_{\mathrm{CD}}(\mathrm{MPa})$ & $\mathrm{E}_{\mathrm{MDflex}} / \mathrm{E}_{\mathrm{MDtensile}}$ & $\mathrm{E}_{\mathrm{CDflex}} / \mathrm{E}_{\mathrm{CDtensile}}$ \\
\hline $844.45 \pm 71.29$ & $605.73 \pm 24.54$ & 0.97 & 1.09 \\
\hline
\end{tabular}

Table 4 : Bending properties.

\begin{tabular}{cccc}
\hline$d x(\mathrm{~mm})$ & 0.1 & 0.005 & 0.0005 \\
\hline$[\mathrm{B}]$ & {$\left[\begin{array}{ccc}-1.543 & 0.773 & 0 \\
0.7738 & -2.620 & 0 \\
0 & 0 & 0.211\end{array}\right] 10^{-3}\left[\begin{array}{ccc}-3.9 & 1.9 & 0 \\
1.9 & -6.6 & 0 \\
0 & 0 & 0.5\end{array}\right] 10^{-4}\left[\begin{array}{ccc}-0.387 & 0.193 & 0 \\
0.193 & -0.663 & 0 \\
0 & 0 & 0.052\end{array}\right]$}
\end{tabular}

Table 5 : Influence of the step integration $d x$ on the global matrix [B] 


\begin{tabular}{cccc}
\hline & Analytical results & $\begin{array}{c}\text { Experimental } \\
\text { results }\end{array}$ & (Anal.-Exp) /Exp. \\
\hline $\mathrm{E}_{\mathrm{MD}}(\mathrm{MPa})$ & 954.34 & 863.05 & $10.57 \%$ \\
$\mathrm{E}_{\mathrm{CD}}(\mathrm{MPa})$ & 552.02 & 554.97 & $-0.53 \%$ \\
$v_{\mathrm{xy}}$ & 0.314 & 0.271 & $15.8 \%$ \\
$\mathrm{G}_{\mathrm{xy}}(\mathrm{MPa})$ & 274.58 & $267.834^{*}$ & $2.51 \%$ \\
$\mathrm{D}_{11}(\mathrm{~N} . \mathrm{mm})$ & 4347.6 & 4537.6 & $-4.1 \%$ \\
$\mathrm{D}_{22}(\mathrm{~N} . \mathrm{mm})$ & 3104 & 3254.84 & $-4.6 \%$ \\
\hline
\end{tabular}

* Obtained by Baum's relation

Table 6 : Comparison between analytical and experimental results

\begin{tabular}{|l|c|c|c|}
\hline N x M & $2 \times 10$ & $4 \times 20$ & $6 \times 30$ \\
\hline d.o.f & 99 & 315 & 651 \\
\hline MD deflection $(\mathrm{mm})$ & 1.3479 & 1.3563 & 1.3562 \\
\hline CD deflection $(\mathrm{mm})$ & 2.4650 & 2.4788 & 2.4787 \\
\hline
\end{tabular}

Table 7- Convergent studies

\begin{tabular}{|c|c|c|c|c|c|}
\cline { 2 - 6 } \multicolumn{1}{c|}{} & $\begin{array}{c}\text { Plate FE } \\
\text { MD }\end{array}$ & $\begin{array}{c}\text { Plate FE } \\
\text { CD }\end{array}$ & $\begin{array}{c}\text { Shell FE } \\
\text { CD }\end{array}$ & $\begin{array}{c}\text { Exper. } \\
\text { CD }\end{array}$ & $\begin{array}{c}\text { Exper. } \\
\text { MD }\end{array}$ \\
\hline $\begin{array}{c}\text { Bending rigidity } \\
\text { (N/mm) }\end{array}$ & 3.68 & 2.017 & 2.47 & $2,29 \pm 0,09$ & $3.39 \pm 0.36$ \\
\hline CPU Time (s) & 0.3 & 0.3 & 3 & $/$ & $/$ \\
\hline
\end{tabular}

Total load is $5 \mathrm{~N}$.

Table 8 : Comparison between FE and experimental results 
